\title{
Einseitige Gesichtsrötung beim Essen
}

Ein gesundes siebenjähriges Mädchen hatte seit der Geburt eine rezidivierende Rötung im Bereich der rechten Wange, die jeweils während des Essens auftrat. Aus der Anamnese war bemerkenswert, dass das Kind mithilfe der Geburtszange zur Welt gebracht wurde. Nach der Konstellation kam nur ein sogenanntes FreySyndrom in Betracht, das auch als gustatorisches Schwitzen oder gustatorische Hyperhidrose bekannt ist. Ursache ist eine Schädigung von Fasern des N. auriculotemporalis (hier offenbar durch die Geburtszange), die bei der Regeneration statt der Glandula parotis fälschlicherweise Schweißdrüsen oder Blutgefäße stimulieren. Es kommt dann zum gustatorischen Schwitzen oder zur gustatorischen Hyperämie. Das Phänomen kann beim Essen, aber auch beim Kaugummikauen, Bonbonlutschen oder Beißen auftreten.
Die Auslösung mittels Verletzung durch die Geburtszange ist sehr selten. Viel häufiger entsteht die Symptomatik durch andere Gesichtstraumen, Operationen, Infektionen oder Parotistumoren. Sie kann mit einer Kontaktdermatitis oder einer Nahrungsmittelallergie verwechselt werden, doch sprechen die völlig normale Hautstruktur und die typische einseitige Verteilung auf die Präaurikulärregion und die Wange dagegen. Die Diagnose der irreversiblen Funktionsstörung ist klinisch zu stellen. Weitere Untersuchungen sind nicht erforderlich. Das sehr beeinträchtigende Syndrom des gustatorischen Schwitzens kann heute mittels lokaler Injektion von Botulinum Toxin A effektiv behandelt werden.

Prof. Dr. med. H. S. FüeßI

- Daniels E,Watchorn R (ella.daniels@nhs.net).Unilateral facial flushing precipitated by eating. BMJ. 2016;352:i1377

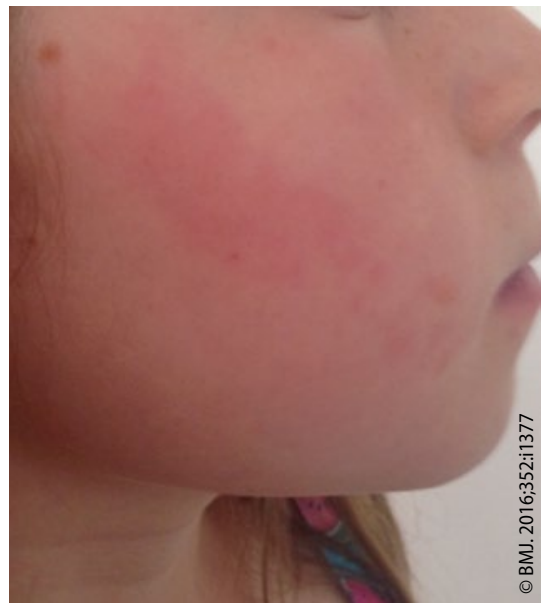

Rezidivierende Rötung bei der Nahrungsaufnahme im Bereich der rechten Wange.

\section{Nadeln gegen menopausale Hitzewallungen?}

\author{
Akupunktur nach den Regeln der traditionellen chinesischen Medizin ist gegen Hitzewallungen \\ in der Menopause nicht effektiver als Scheinakupunktur.
}

_ Seit die Hormonersatztherapie bei menopausalen Hitzewallungen wegen des Brustkrebs- und Herz-KreislaufRisikos nur noch selten eingesetzt wird, sucht man nach Alternativen. 327 Frauen über 40 Jahre mit mindestens sieben mittelgradigen Hitzewallungen täglich wurden in zwei Gruppen mit Akupunktur nach den Regeln der traditionellen chinesischen Medizin und Scheinakupunktur randomisiert. Im Verlauf von acht Wochen erhielten die Patientinnen
Hier steht eine Anzeige.

를 Springer zehn Akupunktursitzungen. In beiden Gruppen ging der entsprechende Score nach acht Wochen um etwa $40 \%$ zurück. Dieser Effekt war bei den drei und sechs Monate nach Beendigung der Studie erfolgten Nachuntersuchungen noch in gleicher Höhe feststellbar

- Ee Cet al. Acupuncture for menopausal hot flashes. A randomized trial. Ann Int Med. 2016;164:146-54

\section{KOMMENTAR}

Ähnlich wie bei den deutschen AkupunkturStudien zum Kniegelenkschmerz zeigte sich, dass Akupunktur ein wirksames Verfahren ist - dessen Wirkung aber ausschließlich auf dem Placeboeffekt beruht. Der vorwissenschaftlich-philosophische Überbau mit Energiebahnen ist dabei überflüssiges Beiwerk. Darauf deuten auch Studien hin, die zeigen, dass die Wirksamkeit des Verfahrens noch erhöht werden kann, wenn der akupunktierende Arzt chinesisch aussieht.

Prof. Dr. med. H. S. Füeß 